\title{
PARTICLE ACCELERATORS AND DISCOVERIES IN ELEMENTARY PARTICLE PHYSICS
}

\author{
Lawrence $W$. Jones \\ Randa 11 Laboratory of Physics, University of Michigan \\ Ann Arbor, Michigan 48109-1120
}

\begin{abstract}
Some discoveries in elementary particle physics are recounted from personal and historical perspective with particular reference to their interaction with particle accelerators. The particular examples chosen include the $\psi / J$, the $\Upsilon$, and the study of nucleon constituents with inelastic electron scattering. Precurser experiments are cited together with the better known discoveries.
\end{abstract}


PARTICLE ACCELERATORS AND DISCOVERIES IN ELEMENTARY PARTICLE PHYSICS

\author{
Lawrence $W$. Jones* \\ Randa 11 Laboratory of Physics, University of Michigan \\ Ann Arbor, Michigan 48109-1120
}

\begin{abstract}
Dr. Month asked me to prepare a talk on high energy physics discoveries and their relationship to particle accelerators. No particular time period was specified; however, high energy physics as a field is less than forty years old. This historical subject was a challenge for me as there was clearly far too much to cover in a comprehensive manner in just one hour. Therefore, I was forced to pick and choose. I ain not an historian of physics, and I have not made a systematic study of the recent history of particle physics. Rather I am a physicist who is now becoming old enough to have "been there" when some of these discoveries were made. I have used as one source for this talk the material contained in a report prepared for a larger document "Physics in the 1980's" edited by Dr. Brinkman. Martin Perl of Stanford has chaired a subpanel on elementary particle physics of that task force, on which I served.
\end{abstract}

I will take two topics from the broad subject matter of particle physics and focus on them: the quark model of hadrons and the quark-lepton interactions as they relate to nucleon structure functions. I will not reach back earlier than the 1960's and I will not try to bring my report up to those most recent experiments which have been the subject of many recent papers, seminars, and lectures. Let me make one warning here: this lecture may be much too elementary for most of you. It is based on the assumption that many of you may be from other fields, or you have been so immersed in accelerator science that you may not have had the opportunity of studying particle physics in depth. We learn by studying history, therefore the objective of a lecture of this character is to observe patterns and to learn from the mistakes and successes of the past in order to assist us in most effectively studying our field and making advances in the future. One thing that has interested me is to observe "precursors" to particular discoveries, that is, to note where there were experiments which preceded the definitive experimental work but in retrospect reported evidence for the same phenomena later announced. I will also depart somewhat from Dr. Month's assignment by identifying instances where accelerators were not used in making important discoveries.

Let me first turn to the subject of the quark model of hadrons. Briefiy recapitulating history, the neutron was dicovered in 1932 and the concept of isotopic spin as applied to nuclear states first appeared in the $1930^{\prime} \mathrm{s}$. In 1946 the charged pion, or pi-meson, was observed in cosmic rays; only a little later the neutral pion was

* Supported in part by the U.S. National Science Foundation. 
identified at particle accelerators. The nucleon $3 / 2,3 / 2$ isobar state, the $\Delta(1238 \mathrm{MeV})$, was first observed by Fermi and his collaborators in the early $1950^{\prime} \mathrm{s}$, and the strange particles, the $\Lambda$ and K-meson, were found in cosmic rays about 1948. So already by the early $1950^{\circ} \mathrm{s}$ there came to be known a large number of meson and baryon states. The Brookhaven cosmotron first turned on in 1952 and the 6.GeV Bevatron at Berkeley came into operation in 1955. 1954 was also approximately the time the bubble chamber was developed as a tool for the study of particle physics, and following the application of the bubble chamber to the beams from the Bevatron and Cosmotron, there developed the virtual explosion in the discovery of particle resonances.

The proliferation of meson and baryon states was one stimulus to the development of the quark mode1. Another independent stimulus was provided by the experiments on elastic electron-proton scattering. In the mid-1950's also, Robert Hofstadter, working with the Stanford electron linear accelerator (at that time with an energy of about $180 \mathrm{MeV}$ ) studied the angular distribution of the elastically scattered electrons from protons. This was the $1950^{\prime} \mathrm{s}$ analog to the classical Rutherford scattering experiment of alpha particles on gold. The consequence of Hofstadter's experiment was that the scattering distribution of the electrons did not follow the distribution expected for a point charge scattering center. Let me reproduce below the summary of the relevant kinematics and related formula.

Consider the Feynman diagram below (time advances to the right in the figure).

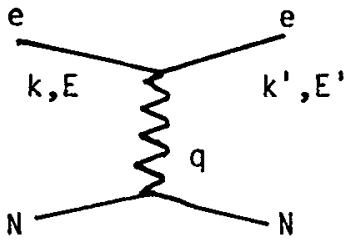

$$
\begin{aligned}
& q=k-k^{\prime} \\
& \tau=-q^{2} / 4 M^{2}
\end{aligned}
$$

The differential angular distribution of the scattered electron is given by

$$
\frac{d \sigma}{d \Omega}=\frac{\alpha^{2}}{4 E^{2} \sin ^{4} \theta / 4}\left(\frac{E^{\prime}}{E}\right) \quad\left[\frac{G_{E} 2+\tau G_{M} 2}{1+\tau} \cos ^{2} \frac{\theta}{2}+2 \tau G_{M}^{2} \sin ^{2} \frac{\theta}{2}\right]
$$

where

$$
G_{E}\left(q^{2}\right)=\frac{1}{\left(1-\frac{q^{2}}{0.71}\right)^{2}} \quad \text { and } \quad G_{E}=\frac{G_{M}}{\mu}
$$


The results are that (in the non-relativistic limit)

$$
\rho(r) \times e^{-r / b} ; \quad b^{2}=1 / 0.71 \text {. }
$$

The consequence of these experiments gave the value for the RMS radius of the proton of about 0.81 fermi. ${ }^{1}$ These data were interpreted by Chew and his collaborators at Berkeley as evidence for a vector meson particle in the nucleon structure, that is, the electron was perceived as radiating a virtual gamma ray which coupled to a vector particle in the field of the nucleon (the neutron or the proton). This vector particle was predicted to be a combination or a mixture of an isoscalar or an isovector particle. Later we came to know these as the $\rho$ and the $\omega$ mesons. The mass of the $\rho$ was predicted by Chew from the electron scattering experiments to be about $450 \mathrm{MeV}$, and indeed several experiments set out to look for this vector meson.2

I am particulariy aware of this bit of history because at Berkeley, Martin Perl and I, working with graduate students, set out to look for such a particle in 1960 by directing a beam of negative pions on a small hydrogen target and looking at the energy and angular distribution of recoiling protons. 3 In order to deduce the mass of the scattered meson state we ran this experiment through a range of incidence pion energies that would be sensitive to the 450 MeV final state meson, but we deliberately avoided extending our incident energies so high that we were above the associated production $(\Lambda-K)$ threshold, because we were concerned that we would not be able to resolve the background from decaying lambdas, and the interpretation of the data would have then been ambiguous. Of course not long afterward, Walker and his collaborators, using a small hydrogen bubble chamber, discovered the $\rho$ at about $760 \mathrm{MeV},{ }^{4}$ whereas our mass search had only extended up to about $695 \mathrm{MeV}$. We thus learned a lesson to never take the theorists too seriously.

Subsequentiy, of course, the $\omega^{0}$ and the $\eta^{0}$ meson were found at Berkeley; anti-proton annihilation produced invariant mass states that were identified with various resonances ${ }^{\text {in }}$ abouf 1961 . In about 1962 the $f^{\circ}$ was observed, later the $\phi, K^{*}$, the $A^{1}$ and others were found as well as the baryon family of particles: the $\Lambda^{*} s$, $\Sigma s$, $\Xi s$, and a whole host of nucleon isobars. ${ }^{5}$ The vast majority of these particles were discovered using liquid hydrogen bubble chambers. Now the bubble chambers did not place intensive or excessive demands on operators of accelerators. They did require clean beams and the requirement on accelerator intensity was primarily to provide adequate fluxes at higher energies of negative kaons and anti-protons. The other requirement (which was easy to fulfill) was fast extraction. The bubble chamber should have a beam pass through it in a time interval no more than tens or hundreds of microseconds prior to flashing the light (to record a photograph). The $\mathrm{K}^{-}$separation from $\pi^{-}$at high energies was a challenging task which was accomplished by electromagnetic separators, devices which are not studied particularly these days. They used a very simple 
concept of crossed electric and magnetic fields and of course the optics of strong focusing lenses. Much of this progress was due to Luis Alvarez who, in the early days of the Bevatron, insisted that the detectors to be used with the new generation of accelerators warranted an investment that was significant, i.e., it should be in some sense proportional to the effort invested in the accelerators. Thus, the Berkeley 72-inch hydrogen bubble chamber was an enormously large and expensive detector in the context of those years and represented an investment on the order of magnitude of $10 \%$ of the investment of the Bevatron itself, where by "investment" I could equally well consider either money or people or laboratory resources.

In the early 1960 's the quark model as a framework for understanding the rapidly multiplying numbers of mesons and baryons was put forth independently by Murray Gel1-Mann ${ }^{6}$ and by George Zweig. 7 As you may know, Gell-Mann's work was published promptly, whereas Zweig's original paper is a CERN report, and (as I have been told) a report which the CERN theoretical group did not feel should be submitted for publication. In any case, the concept here was that all known particles, which then included strange and nonstrange mesons and baryons, might be composed of three fundamental objects or "quarks" (Zweig called them aces). We now refer to the se quarks as the up, down, and strange quarks (the $u, d$, and s) with a one-third or two-thirds integral charge, one-third baryon number, and half-integral spin. Gell-Mann held from the outset that these might be only mathematical constructs and not physical objects. On the other hand, other theorists felt very strongly that if quarks had meaning in the model, they should be physical objects which could be produced and detected in high-energy collisions. Richard Dalitz was a primary proponent of this point of view. One of the turning points in the development of the quark model was the prediction that there was an undiscovered member of a baryon decouplet whose other nine members were the $\Delta^{\prime} s$, the $\Sigma^{*_{1}} s$, and the $\Xi^{*^{\prime}} s$, as shown in the illustration (Figure 1). The missing member was the $\Omega^{-}$, the baryon composed of three strange quarks with a spin of $3 / 2$ but in only the negative charge state. ${ }^{8}$ In 1964 this particle was in fact discovered at Brookhaven, again using a hydrogen bubble chamber and a $5-\mathrm{GeV} / \mathrm{c} \mathrm{K}^{-}$beam. ${ }^{9}$ The event was identified as a consequence of the observation of two kaons in the final state; on close examination the event contained the cascade of an $\Omega^{-}$going to a $\Xi^{0}$ which in turn decayed to a $\Lambda^{0}$ which then decayed to a $\pi^{-}$and a proton, all within the bubble chamber.

Now my first example of a precursor is to reference here a 1973 paper by Luis Alvarez entitled "Certification of Three 01d Cosmic Ray Emulsion Events as Omega Minus Decays and Interactions".10 I quote here verbatim the abstract of that paper.

"In the preaccelerator years when large stacks of emulsion were exposed to cosmic rays at high altitude, three events were found in which $\mathrm{K}^{-}$mesons were emitted from slowly moving particles. The $\Omega^{-}$is the 
(a)

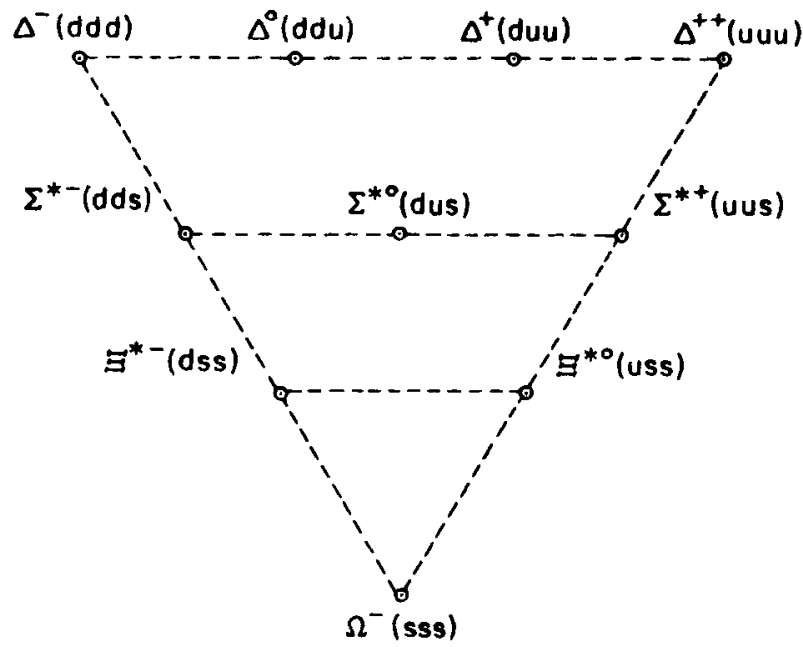

(b)

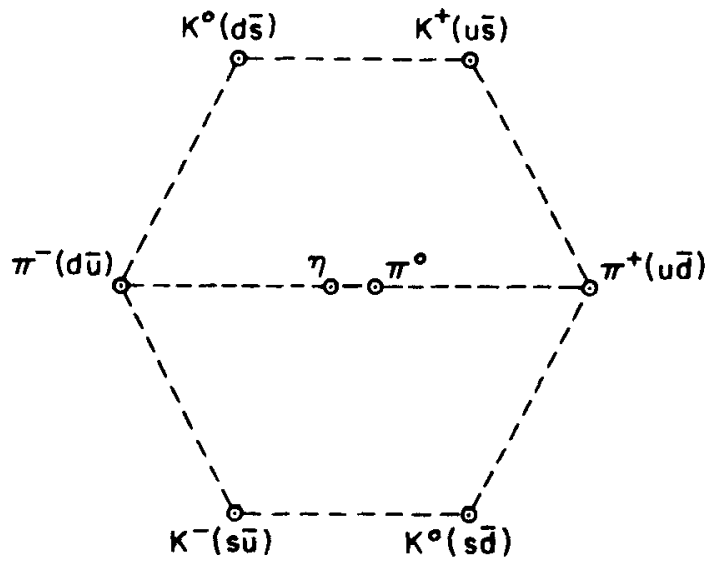

Fig. 1. The $\Omega^{-}$was predicted by Ge11-Mann and was subsequently discovered in the bubble chamber experiments. Figure (a) shows how the delta, sigma-star, xi-star, and omaga family of hadrons are made out of three quarks; (b) shows how the meson family which contains the pion and kaon are made of a quark and an antiquark. The positive pion, $\pi^{+}$, and the positive kaon, $\mathrm{K}^{+}$, have different properties because the $\pi^{+}$consists of an up (u) quark and a down antiquark $(\bar{d})$ while the $K^{+}$consists of an up quark (u) and a strange antiquark $(\bar{s})$. The $n$ and $\pi^{0}$ are made of combinations of $u \bar{u}, d \bar{d}$, and $s \bar{s}$ quarks. 
only presently known particle that can give rise to a $\mathrm{K}^{-}$when moving at nonrelativistic speed, but none of the three events has until now been clearly identified as an $\Omega^{-}$. One of the cosmic ray events (Eisenberg, 1954) has been incorrectiy intrepreted as an $\Omega^{-}$decaying in flight; it is now shown to be an interaction in flight of an $\Omega^{-}$with a silver nucleus. The second event is a clear cut example of an $\Omega^{-}$decaying in orbit bound to an emulsion nucleus. The third event is quite complicated, but can be unambiguously attributed to the decay of an $\Omega^{-}$atomically bound to an $N^{14}$ nucleus followed by a collision of the daughter $\Lambda$ with the $N 14$, in which the compound system then fragments into $\Lambda \mathrm{Cl}^{13}+\mathrm{p}+n$. The mass of the $\Omega^{-}$determined by each of the last two events (Fry et al., 1955) agrees closely with the mean of all bubble chamber events."

The clinching arguments for the validity of the quark model really came with the discovery in 1974 of the $\Psi$ or J particle. This remarkable period in particle physics included the discovery at the Brookhaven National Laboratory of the $J$ by a group directed by Samuel Ting. The $J$ particle was produced in proton-nucleon collisions from the Brookhaven AGS and detected through its decay into an electron-positron pair.11 Nearly simultaneousiy, there was the observation at the SPEAR storage ring at SLAC of electron-positron anninilation into the $\Psi$ particle, in turn producing lepton pairs or hadron pairs in its decay.12 This experiment was led by a group under the direction of Burton Richter. Among the properties of $\Psi / J$ particle were the fact that it was more massive than any other previously observed hadronic state of baryon number zero. It was about 1000 times more stable, i.e., displayed a narrower width than any massive mesonic state might be expected to from previous experience, and its quantum numbers appeared to be the same as the $\phi^{0}$, the $\omega^{0}$, and the $n^{0}$ mesons. (See Figure 2.)

Some background remarks are appropriate here. At Stanford in the $1960^{\prime} \mathrm{s}$ Gerald $\mathrm{K}$. $0^{\prime} \mathrm{Ne} i 11$ had led a group in constructing a pair of $500 . \mathrm{MeV}$ electron storage rings to provide electron-electron collisions for the first time. SPEAR was built as an electronpositron colliding beam ring under Richter's direction. Other $\mathrm{e}^{+} \mathrm{e}^{-}$rings were built at Frascati in Italy, at Orsay in France, and at Novosibirsk in the USSR. In the late 1960's and earlier 1970's the electron-positron anninilation to produce vector mesons such as the $\rho^{0}, \omega^{0}$, and $\phi^{0}$ had already been studied. However, the 1974 observation of the $\Psi$ as a very narrow resonance in the $\mathrm{e}^{+} \mathrm{e}^{-}$ annihilation cross section was the first breakthrough discovery of the colliding beam technique. Subsequently, a rich spectroscopy developed in the $\Psi$ system where the singlet and triplet $p$ states corresponding quite identically to the spectral states of positronium were observed and studied. Of course the $\Psi$ has since been understood as the vector meson whose components are the charm quark and its anti-quark. (See Figure 3.) 


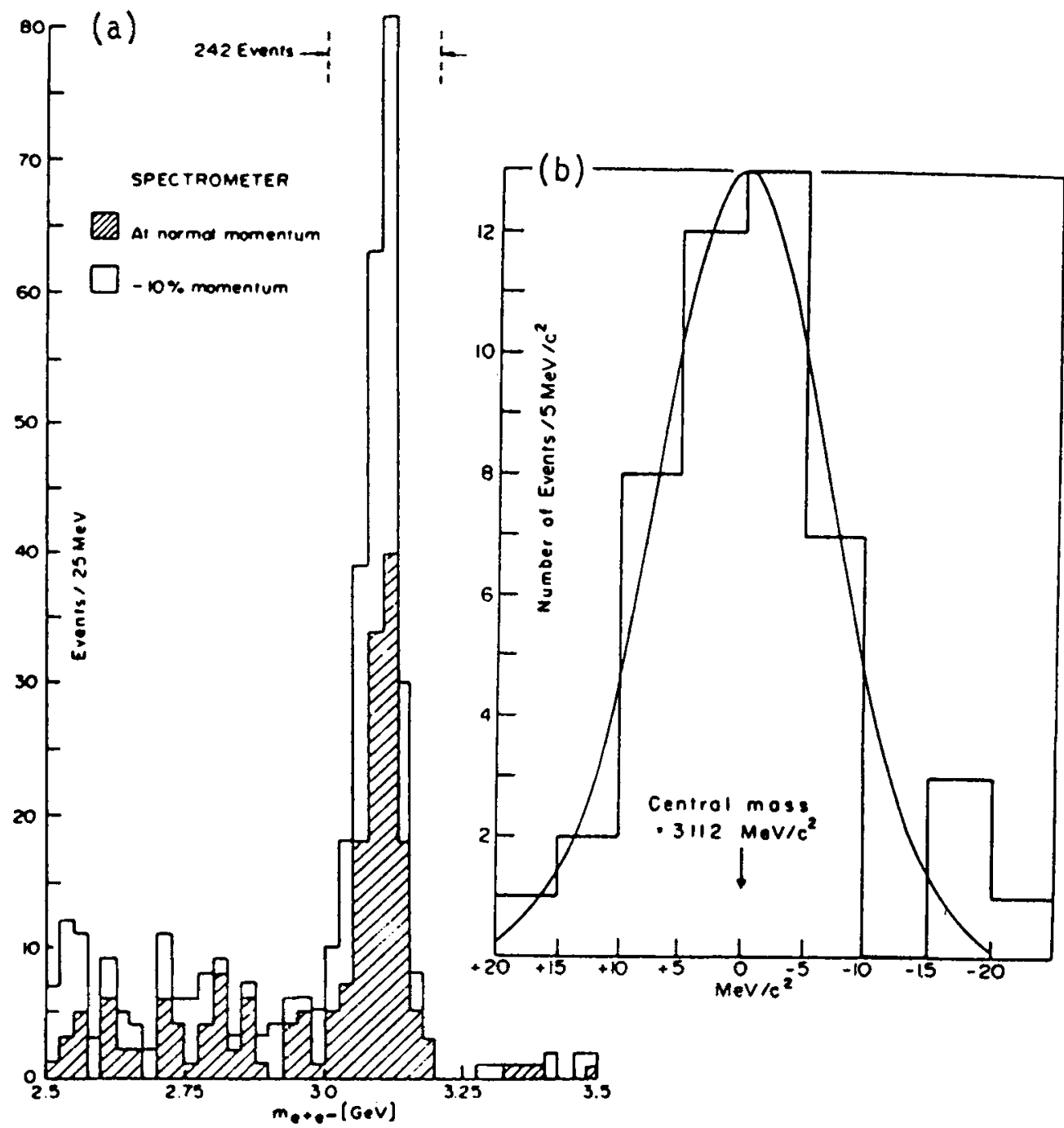

Fig. 2. Data from reference 11 on $e^{+} e^{-}$mass spectrum from the Brookhaven AGS; this was the experiment that first identified the J-meson in strong interactions. (a) Mass spectrum for events in the mass range $2.5<\mathrm{m}_{\mathrm{ee}}<3.5 \mathrm{GeV} / \mathrm{c}$. The shaded events correspond to those taken at the normal magnet setting, while the unshaded ones correspond to the spectrometer magnet setting at $10 \%$ lower than normal value. (b) The measurement of the width of the $\mathrm{J}$. The width is shown to be less than $5 \mathrm{MeV}$. 

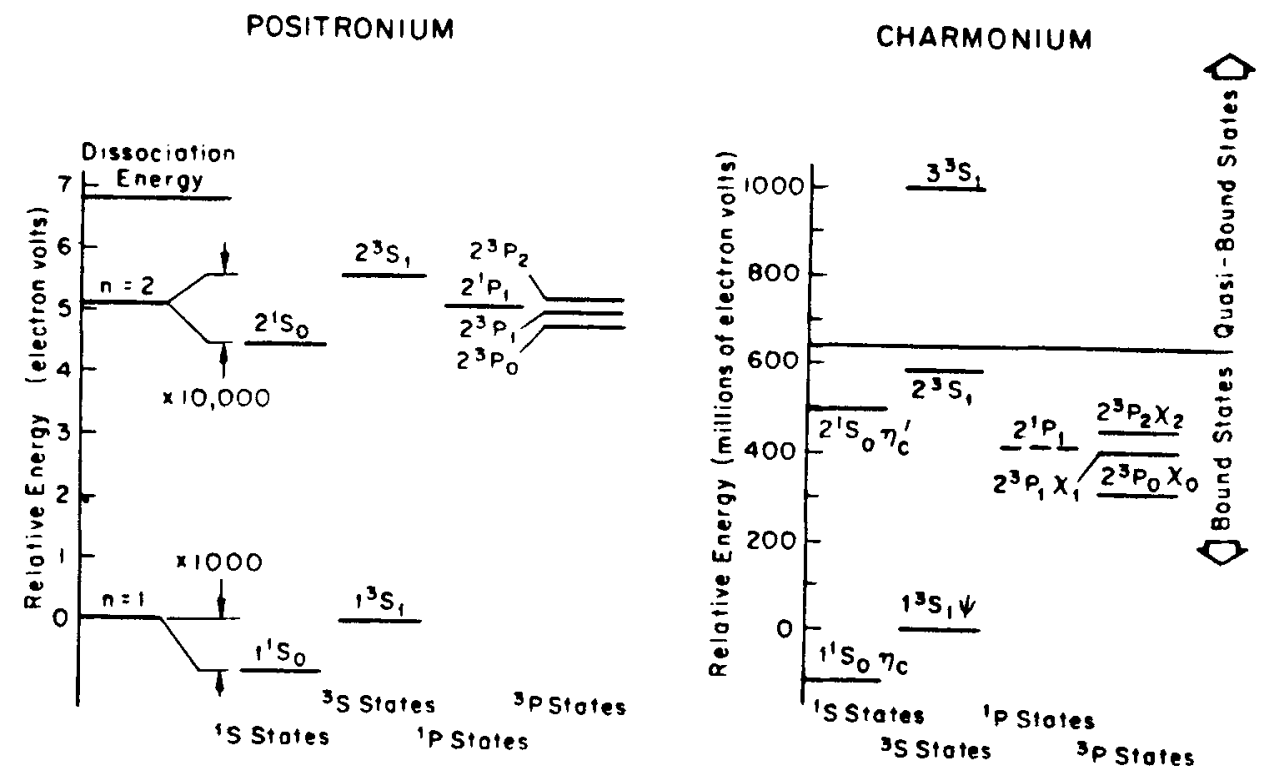

Fig. 3. The spectrum of energy states is similar in positronium and charmonium, but the scale of the energy differences in charmonium is greater by a factor of roughly 100 million. All energies are given with reference to the $1^{3} S^{1}$ state. At 6.8 electron volts positronium dissociates. At $633 \mathrm{MeV}$ above the energy of the $\psi$ charmonium becomes quasi-bound because it can decay into $D^{\circ}$ and $\bar{D}^{\circ}$ mesons.

The experiment at Brookhaven under Ting's direction was very different and totally unrelated to the Stanford work. Ting had earlier studied electron-positron final states of vector mesons working with the 6-GeV electron synchrotron and later with the DORIS electron-positron storage ring at DESY in West Germany. In his Brookhaven experiment he directed a $30-\mathrm{GeV}$ proton beam at a target and looked at the invariant mass spectrum of electron-positron pairs produced. This required a double-armed spectrometer with magnetic analysis, precise particle tracking, and special Cherenkov counters in each leg. He observed a remarkable sharp spike in the invariant mass of the electron-positron pairs resulting from these collisions and identified this particle as the $\mathrm{J}$ meson. Of course, it was later recognized that the $\Psi$ and the $J$ are identically the same state. 
Once again it is interesting to look at precursors or experiments which anticipated these results. In 1970 Leon Lederman and collaborators undertook an experiment to study the invariant mass spectrum of muon pairs produced by protons on a uranium target at the Brookhaven AGS.13 Their experiment differed from Ting's in that the muon energies were determined by a coarse range measurement using stepped absorbers, and the muon angles by a modest number of counter telescopes, so that the resolution in invariant mass of the dimuon pairs was rather modest. They reported no clear evidence of new states although their data does show a shoulder at the mass that later proved to be the invariant mass of the $\psi / J$ state. The conclusion of the authors in their paper was that, "As seen both in the mass spectrum and the resulting cross section $\mathrm{do} / \mathrm{dm}$ there is no forcing evidence of any structure." This paper was the first report of the process which later became understood by Drell and Yan and studied as the Dre11-Yan process.14 However, Lederman's group was not confident of the physics of the process and was uncertain whether a departure from a smooth curve warranted interpretation as a new state. As a pioneering work this earlier dilepton experiment was totally successful; however, I am certain that the authors have since kicked themselves for missing the dramatic discovery which was later made by Ting's group as a consequence of much finer resolution. (See Figure 4.)

Fig. 4. Cross section versus dimuon invariant mass from reference 13. This experiment clearly observed the $\psi / J$ but lacked sufficient energy resolution and knowledge of the Drell-Yan process to identify it as a new object.

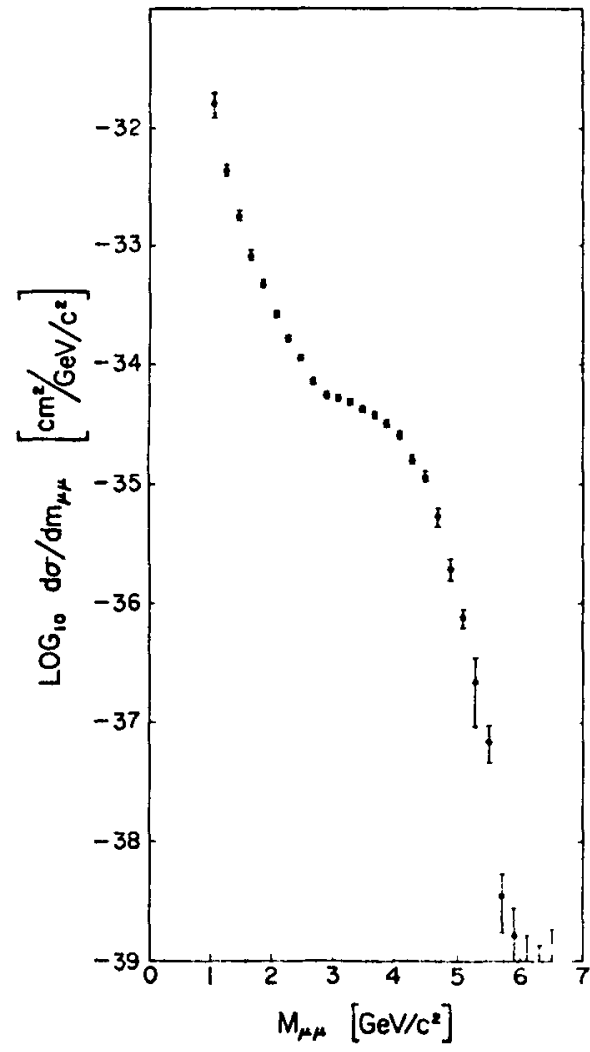


Subsequently, of course, the experiments at Stanford and other $e^{+} e^{-}$colliders observed the $D$ meson, the $D^{\star}$, the $F$, etc.; all mesons containing the charm quark. The study of these charmed mesons was also carried out at hadron machines, although there it proved much more difficult. There were many, many experiments at Fermilab looking for the $D$ meson through the mid-1970's, none of which was particularly successful. However, again there was a precursor. In 1970 a Japanese group, flying emulsion stacks in aircraft, reported the observation of short-lived particles produced in high energy proton collisions in emulsions. In particular, a paper presented in 1971 by Dr. Niu at the 12th International Cosmic Ray Conference in Hobart, Tasmania, reported a very interesting event, where two shortlived particles were produced in the same collision.15 one of the particles decayed in part into a neutral particle which gave rise to two electromagnetic showers with a separation and energies such that the neutral particle was clearly identified as $\pi^{\circ}$. The lifetime of the particles appeared to be of the order of $10^{-13}$ seconds and in retrospect it is quite clear that what $\mathrm{Niu}$ had observed was a pair of $D$ mesons produced in the cosmic-ray collision. Although the incident energy of the cosmic ray was very much higher than the Tevatron beam energy, the analysis of the final state did not permit particle identification (other than the $\pi^{\circ}$ ) so that a determination of the invariant masses was not possible. Niu reported several other interesting events, most of which contained single short-lived final-state particles. Although the cosmic-ray literature is not read as commonly by particle physicists and although Niu's knowledge of the particles he reported was necessarily $7 i m i t e d$, in retrospect $I$ believe we must credit him and his group with the first documented observation of a charmed meson. (See Figure 5.)

The great success of the experiments of Ting and Richter stimulated further work on studies of the Drell-Yan process and of $e^{+} e^{-}$ collisions. Having found one heavy quark, the search was on to find yet more massive states. Lederman's group at Fermilab set up a spectrometer system to look for high mass states decaying into muon pairs or electron-positron pairs over the invariant mass range of $2-1 / 2$ to $20 \mathrm{GeV}$ using $400-\mathrm{GeV}$ protons on a beryllium target. In a Physical Review Letter published in 1976, Lederman's group reported a clustering of 12 electron-positron pairs with an invariant mass between 5.8 and $6.2 \mathrm{GeV}$ which they said suggested that the data contained a new resonance at about $6 \mathrm{GeV} .16$ However, they stopped short of making a dramatic claim for a new particle. The word of this indicated resonance spread rapidly and others were quick to follow.

Another Fermilab group working in the meson area set out to confirm this discovery and in a 1976 publication claimed confirming evidence. The abstract from their paper states:17

"In a simple search for muon pairs directly produced in proton-nucleon collisions at $300 \mathrm{GeV}$ performed with two range telescopes looking at a beam dump we observed the $\Psi$ ( $3.1 \mathrm{GeV}$ ) and have an indication for a structure around $m_{\mu}{ }^{2}=36 \mathrm{GeV}^{2}$." 


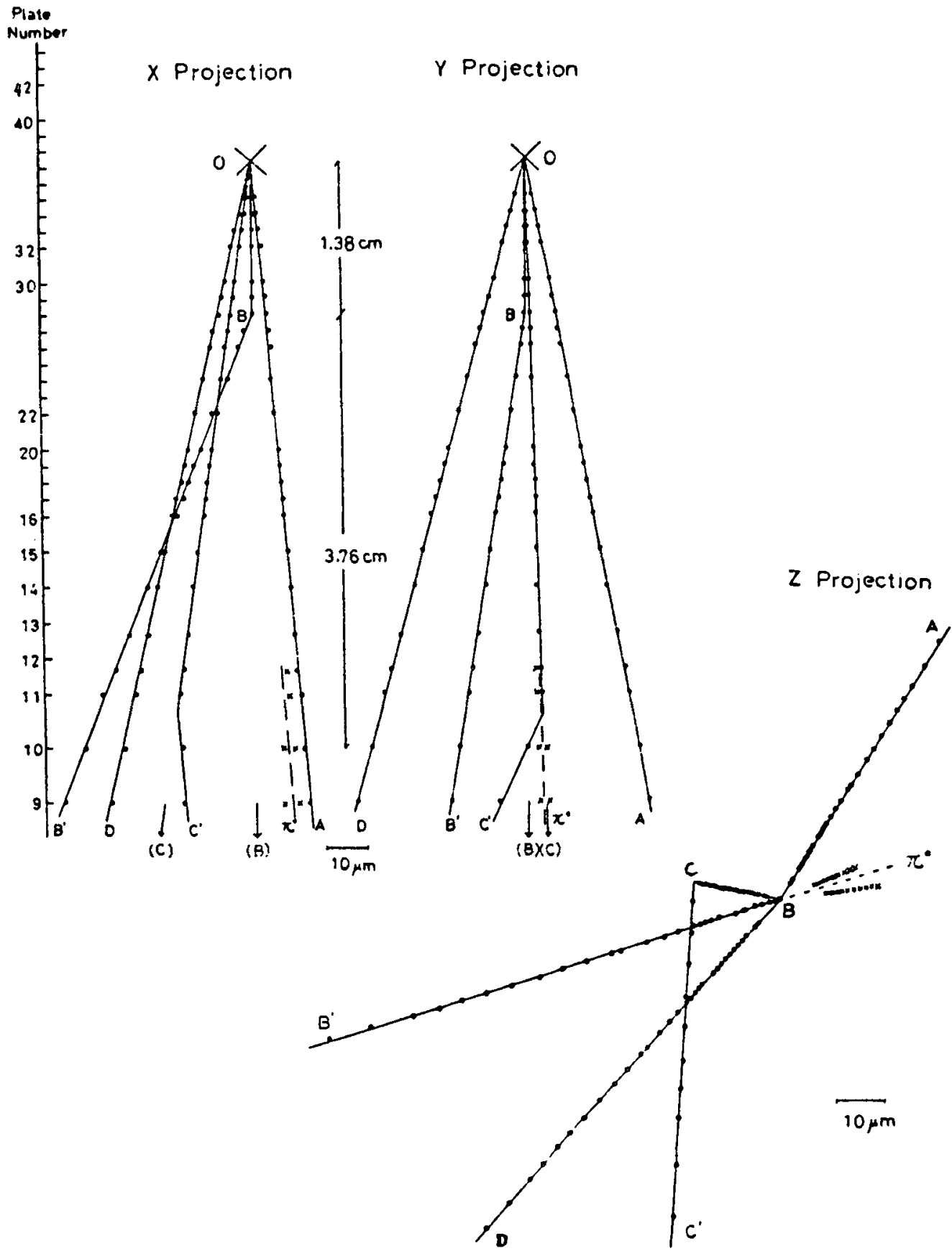

Fig. 5. Reconstruction of an emulsion event from a cosmicray exposure by Niu et al. (reference 15) with first observed example of associated production of charmed particles. 
Their evidence was statistically no better than Lederman's and their mass resolution somewhat worse. Subsequent experiments, of course, failed to confirm the 6-GeV state which Lederman had already tentatively dubbed the upsilon. In 1977, Lederman's group in looking at muon pairs did a considerably more sensitive study and clearly observed the state now universally recognized as the upsilon $(T)$, the state with a mass of $9.4 \mathrm{GeV}$ decaying into lepton pairs.18 The electron-positron colliders, as well as further experiments with hadron accelerators have now established the spectroscopy of the upsilon and there is no question that it is a resonance of the $b$ and $\bar{b}$ quark, hence a new quark had been discovered. (See Figure 6.)

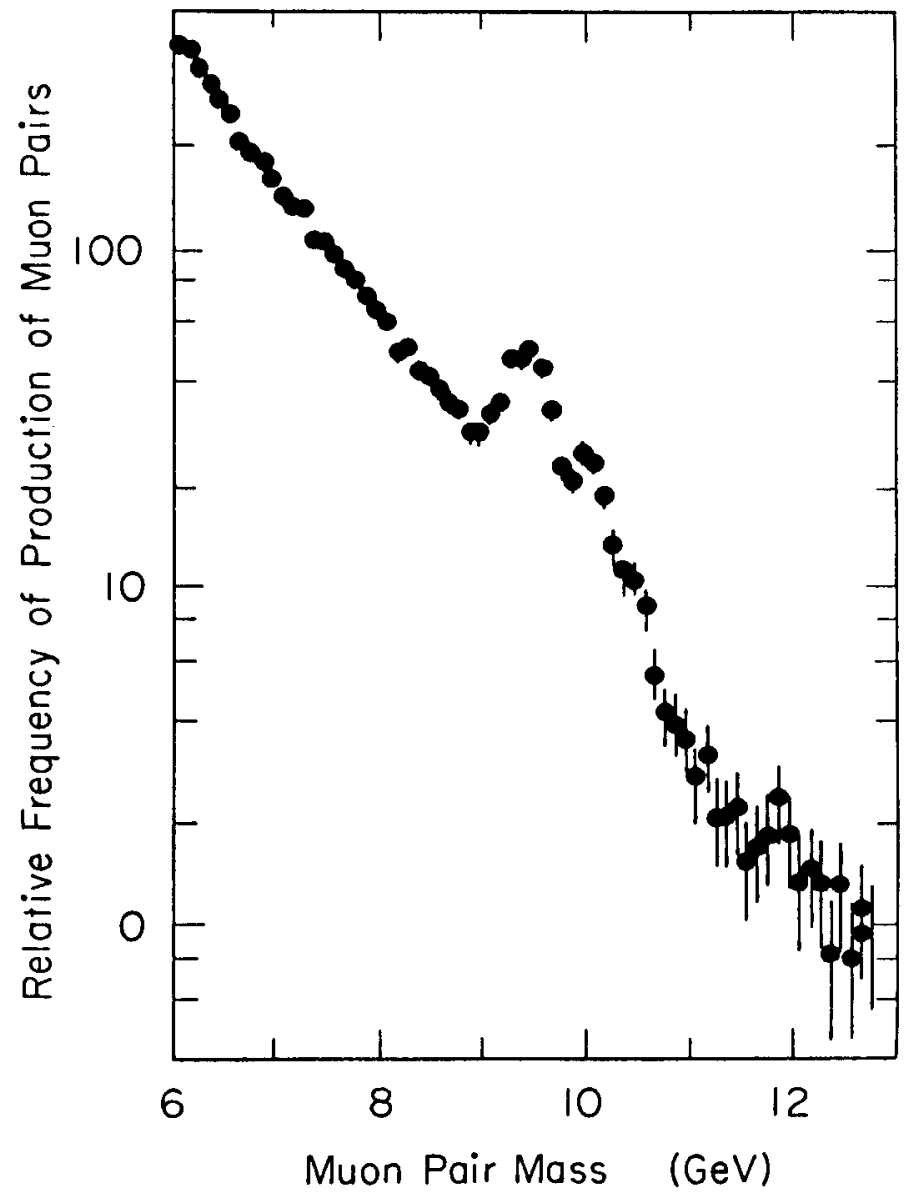

Fig. 6. The upsilon was discovered in 1977 by studying the production of muon pairs or electron pairs in proton collisions. Here the relative frequency of production of muon pairs is shown to decrease as the muon-pair mass increases. The bump in the curve at $9-10 \mathrm{GeV}$ is due to the upsilon. 
This discussion of new states of matter would not be complete without reference to the experiments at Stanford in 1975 which resulted in the discovery of the $\tau$ lepton. In studying data from the Mark I detector at SPEAR, Martin Perl and his group observed some final states which consisted only of an electron and a muon but with considerable missing energy and missing momentum.19 These they concluded were compatible with the interpretation that a pair of massive leptons was produced, each of which decayed leptonically to an electron or a muon and neutrinos. The events only occurred above a threshold of about $3.5 \mathrm{GeV}$ total energy and the $\tau$ mass was subsequently confirmed to be $1784 \mathrm{MeV}$. Consequently, the three generations of matter as they are now understood can be represented as shown on Table I. The top quark is generally conceded to exist although its mass is still uncertain. The $\tau$ neutrino has not been directly observed but it is also generally believed to exist.

TABLE 1. Our present knowledge of the lepton and quark families of particles.

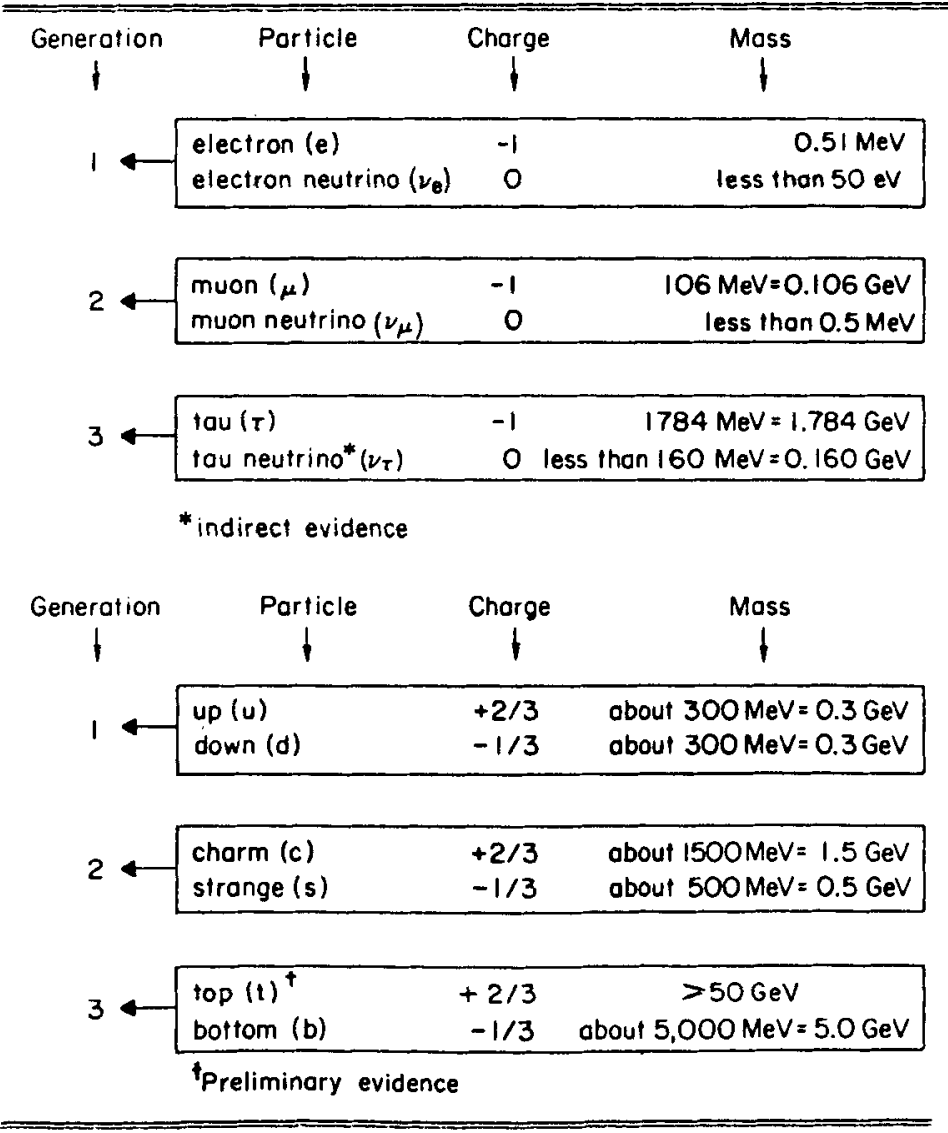


It is amusing in this context to consider the complexity of matter as it has been understood by man down through the ages. A philosophical simplification was made by the Greeks when they believed that all of nature could be understood in terms of earth, air, fire, and water; just four fundamental constituents. However, the alchemists of the middle ages found that a great degree of complexity was necessary; they identified many pure substances such as sulfur, mercury, salt, and so forth which could not be fittedinto the earth, air, fire, and water scheme easily. A simplification came in the 19th century with the atomic theory of matter and the understanding that the larger number of chemical compounds were made of a somewhat smaller number of elements. This understanding reached its peak in a philosophic sense with the Mendeleev Periodic Table and the understanding of the 92 elements. Toward the end of the 19th century the electron was discovered and in the early 20 th century the nuclear atom was understood. For a time there was the appealing concept that all of the elements might be composed of a cloud of electrons of varying numbers corresponding to the chemical element and a heavier nucleus which might be composed of only protons and electrons. However, this model had difficulties with the uncertainty principle and spin; and the discovery of the neutron greatly improved our understanding of the nucleus but added a new particle to our stable. The complexity of nature became greater with the discovery of mesons and during the 1950's became very confused with the strange particles, hyperons, and the proliferation of meson and baryon states. The simplification brought about by the quark model in the 1960 's made it appear that perhaps we only needed a few constituents after all. Now these few constituents have increased to the particles of Table $I$, and we now have six leptons and six kinds of quarks, each of the quarks to come in three colors and each particle with its anti-particle. The fascinating philosophical question at this point is: is this the end? Are these indeed the fundamental constituents of nature; no more and no less? Or might there be yet heavier quarks and heavier leptons; might there be four or five generations? On the other hand, is it possible that all quarks are in turn composed of more fundamental, simpler constituents and only the different configuration or quantum states of these constituents determines the quark species? $0 f$ course, only time and further exploration will answer that question. (See Figure 7.)

I feel it appropriate to remark briefly here that we have searched very diligently for free quarks and it is useful to include here a measure of the limits of the production of free quarks that have been established from various accelerator experiments over the years. 20 The concept of quark confinement is now confortably accommodated by the contemporary theoretical understandings of quantum chromodynamics. Nevertheless, we should recognize that it was forced on theorists earlier when evidence for free quarks simply was not forthcoming from experiments. The positive evidence for free quarks had come from the Fairbank experiments at Stanfor $\mathrm{d}^{21}$ and it is my understanding that these may now be open to some question. (See Figure 8.) 


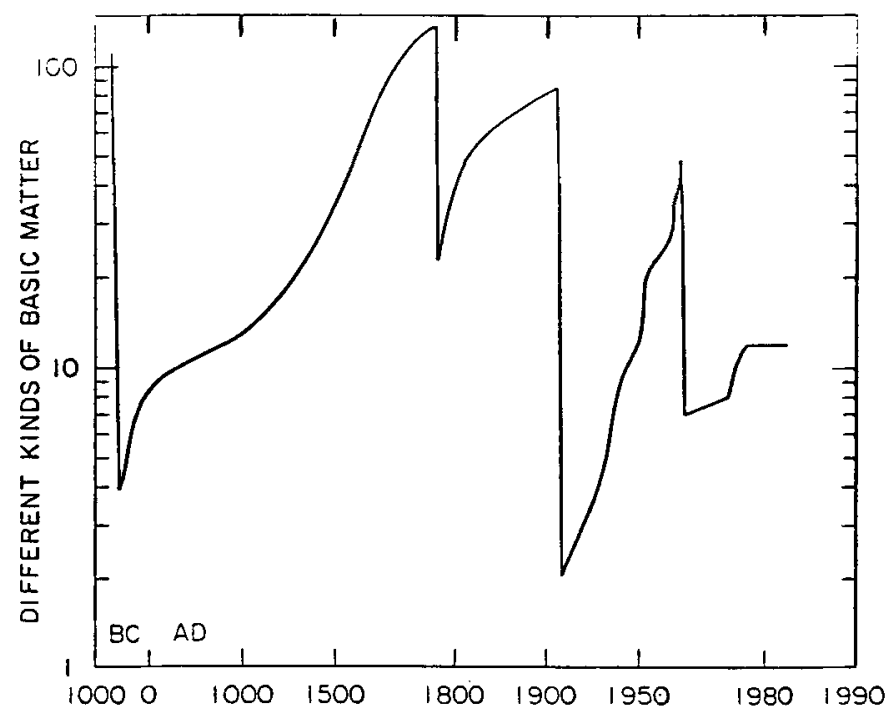

Fig. 7. Mankind has always tried to explain the world as made up of a limited number of different kinds of basic matter. Until a thousand years ago, the basic types of matter were considered to be earth, air, fire, water, et cetera. About 1900 the basic types of matter were thought to be the almost 100 different chemical elements. At present we believe there are about a dozen types of basic matter, namely the leptons and the quarks.

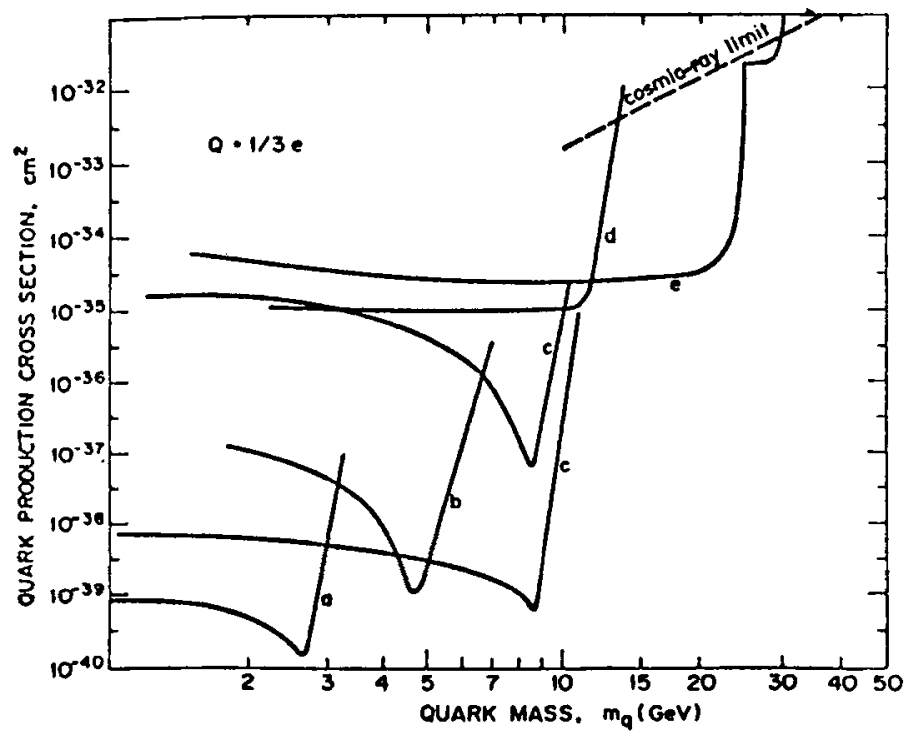

Fig. 8. The upper-limit cross section for production of quarks of charge $-1 / 3$ e as a function of quark mass from experiments at various particle accelerators and cosmic rays. The different experiments are indicated by letters: (a) CERN 28-GeV proton synchrotron; (b) Serpukov 70-GeV proton synchrotron; (c) and (d) Fermi National Acce 1erator Laboratory synchrotron; (e) CERN intersecting storage rings. 
Let me now turn to the second thread in my discussion: the concept of deep inelastic lepton scattering. The finite size of the proton as revealed by electron scattering in the 1950's was one of the hints that protons might have an underlying structure and that the proton might not be a fundamental particle. Later, the inelastic scattering of electrons on protons, where the proton was broken up into a number of hadrons, resulted in explicit evidence that the constituents of the protons might be point particles.

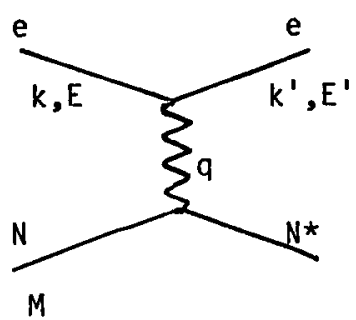

The double-differential cross expressed in terms of structure related $F_{1}$ and $F_{2}$, which characterize

$$
\begin{aligned}
\nu & =E-E^{\prime} \\
y & =\left(E-E^{\prime}\right) / E \\
x & =-q^{2} / 2 M_{v}=+Q^{2} / 2 M_{\nu} \\
Q^{2} & =-q^{2}
\end{aligned}
$$

sections, $d^{2} \sigma / d E^{\prime} d \Omega$, can be functions, $W_{1}$ and $W_{2}$, and the the quark distributions in the nucleon:

$$
\frac{d 2 \sigma}{d E^{\prime} d \Omega}=\frac{\alpha^{2}}{4 E^{2} \cdot \sin ^{4} \theta / 2} \quad\left[W_{2}\left(\nu, q^{2}\right) \cos ^{2} \quad \frac{\theta}{2}+2 W_{1}\left(\nu, q^{2}\right) \sin ^{2} \frac{\theta}{2}\right] .
$$

Experimentally, $u W_{2}$ is seen to be almost independent of $q^{2}$, which suggested in about 1968 that the proton was constructed of point-like constituents.

Currently we have three sources of information on the form factor of protons and neutrons, that is, on the distribution of quark momenta in the proton and neutron. The oldest data come from the Stanford Linear Accelerator experiments on electron-nucleon deep inelastic scattering. 22 This is probably the most important noncollider physics to come from SLAC. Muon beams at Fermilab and CERN have also been used to determine form factors by deep inelastic scattering of muons, where the interpretation of physics is really quite similar to the electron case.23 Neutrino beams at Fermilab and CERN have been used as well to probe the form factor, although in this case not through the exchange of a virtual photon but through the exchange of an intermediate vector boson.24 The neutrino experiments determine the form factor in a rather separate way but the results, in fact, totally confirm the leptonic experiments. It is worth noting that these neutrino experiments at the hadron accelerators have placed the greatest demands on the proton beam intensity of any part of the experimental programs at CERN and Fermilab. As the experiments have become better it is observed that the strict scaling is not maintained and that the form factor determination does depend weakly on $q^{2}$. This departure from scaling is now understood in terms of quantum chromodynamics. (See Figure 9.) 


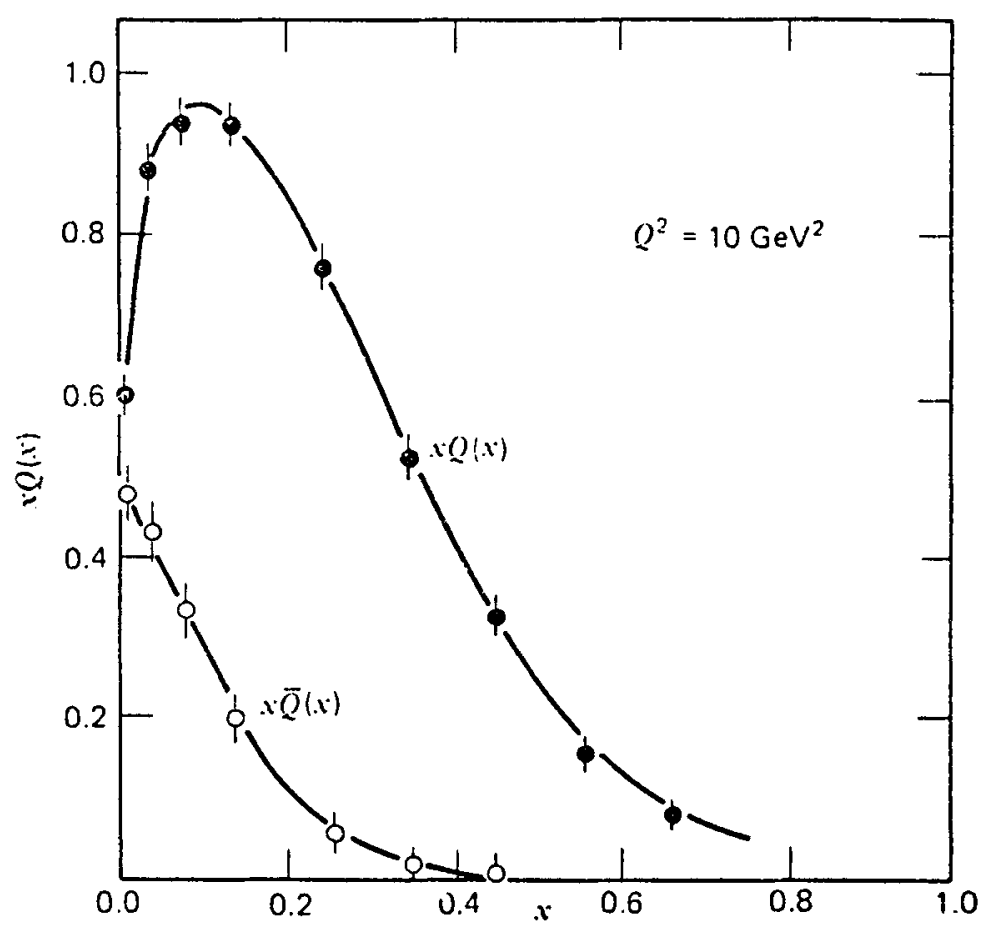

Fig. 9. Quark and antiquark momentum distributions in a nucleon as measured at CERN and the Fermi Laboratory. The experiments reveal that only about half the proton's momentum is carried by quarks. We have associated the remainder with the gluon constituents.

I might summarize briefiy some of the spinoffs from the lepton-nucleon deep inelastic experiments beyond the simple determination of form factors. First, there is a scaling violation.22,23,24 This can be understood by remarking that the quarks with large values of Feynman - $x$ radiate gluons and this softens their scattering contribution.* A second important set of data has clearly identified the electroweak mixing through the parity nonconservation in electron-deuteron scattering using polarized electron beams. 25 A third interesting set of data has come from muon-nucleon scattering, where it is observed that the form factor of quarks determined from muons scattering on an iron nucleus is different from that from scattering on free hydrogen.26 One might have expected that the distribution of the quark velocities in a heavy nucleus might be broader than in hydrogen due to folding of the form factor for a free proton together with the Fermi motion of that proton in the nucleus. In fact, it is observed that the form

"The variable $\mathrm{x}$ defined by Feynman is the fraction of the proton's momentum carried by the constituent quark or nucleon. 
factor is somewhat narrower, as might be understood simplistically from quantum mechanics of a larger confining box and a broader determination of position or a greater uncertainty of position corresponding to tighter distribution in momentum. This EMC effect (as it's called from the European Muon Collaboration) is still a subject of intense study and is very interesting to the nuclear physicists as well as to particle physicists. The Drell-Yan process can also be used to study form factors, and indeed an experiment in which I was involved at Fermilab using proton collisions with tungsten made a very careful determination of form factors from the study of muon pairs.27 The upsilon was also observed in this experiment aithough less clearly than by the Lederman group because of a solid iron spectrometer and the consequent fuzzing of the muon momentum resolution from multiple scattering.

Let me summarize briefly. To be sure, we have only scratched the surface in my simplistic historical summary. We have ignored all hadron dynamics, all of the beautiful studies of the K-meson system with CP violation, etc., and we have ignored almost all of the excitment of electroweak mixing and the other work which had led up to the understanding and later discovery of the intermediate vector bosons. I hope that I have given enough to convey several points, however. One, to emphasize that the threads of our knowledge are interpenetrating, there is a unity in particle physics, and so very often experiments directed at one area find interesting application in understanding other areas. Second, accelerators are used in a wide variety of modes often unanticipated when the machines thenselves were built. Third, energy is usually, although not always, important; it has often been cited that the Ting experiment which discovered the J-particle might have been done fifteen years earlier at the AGS and also could have been done more easily and earlier than 1974 at the Fermilab higher energy accelerator. Fourth, intensity is sometimes critica1, a though the ingenuity of the experimenters, and the reliability and stability of the accelerator, are perhaps more important. Finally, there are often precursors for discoveries. These precursors generally lack either statistics or resolution or a sufficient theoretical understanding of the background phenomenon to have made the definitive experimental statements. And indeed, there are also occasional mistakes. I hope these perspectives might be interesting and might find some application in our thinking about future experimental physics and particle accelerators.

\section{REFERENCES}

1. R. Hofstadter, Rev. Mod. Physics 58, 214 (1956): Annu. Rev. Nucl. Sci.

2. W.E. Frazer and J.R. Fulco, Phys. Rev. 117, 1609 (1960).

3. C.C. Peck, L.W. Jones, and M.L. PerT, Phys. Rev. 126, 1836 (1962).

4. A.R. Erwin, R. March, W.D. Walker, and E. West, Phys. Rev. Lett. 6, 628 (1961). 
5. See, for example, Rev. Mod. Phys. 56, 2, II (1984). "Review of Particle Properties."

6. M. Gel1-Mann, Phys. Lett. $\underline{8}, 214$ (1964).

7. G. Zweig, CERN-TH 412 (1964) (unpublished).

8. M. Gel1-Mann in Proceedings of the International Conference on High-Energy Nuclear Physics, Geneva, 1962, p. 805.

9. V.E. Barnes et a1., Phys. Rev. Lett. 12, 204 (1964).

10. L.W. A7varez, Phys. Rev. D8, 702 (1973).

11. J.J. Aubert et a1., Phys. Rev. Lett. 33, 1404 (1974).

12. J.E. Augustin et a1., Phys. Rev. Lett. 33, 1406 (1974).

13. J.H. Christenson et a1., Phys. Rev. Lett. 25, 1523 (1970).

14. S. Drell and T.-M. Yan, Phys. Rev. Lett. 25, 316 (1970).

15. K.C. Niu, E. Mikumo, and Y. Maeda in Proceedings of the 12th International Cosmic Ray Conference, Hobart, Tasmania (1971); K.C. Niu, E. Mikumo, and Y. Maeda, Prog. Theor. Phys. 46, 1644 (1971).

16. D.C. Hom et a1., Phys. Rev. Lett. $\underline{36}, 1236$ (1976). 17. D. Eartly, F. Giacome11i and K. Pretzl, Phys. Rev. Lett. 36,
$1355(1976)$.

18. S. Herb et al., Phys. Rev. Lett. 35, 252 (1977).

19. M.L. Perl et a1., Phys. Rev. Lett. 39, 1489 (1975).

20. L.W. Jones, Rev. Mod. Phys. 49,717 (1977).

21. G.S. LaRue, J.D. Phillips, and W.M. Fairbank, Phys. Rev. Lett. 46, $967(1981)$.

22. A. Bodek et a1., Phys. Rev. D20, 1471 (1979).

23. A.R. Clark et a1., Phys. Rev. Lett. 51, 1826 (1983).

24. H. Abramowicz et a1., Zeit. Phys. C17, 283 (1983).

25. C. Prescott et a1., Phys. Lett. $\underline{84 B}, 524$ (1979).

26. J.J. Aubert, Phys. Lett. 123B, 275 (1983); A. Bodek et al., Phys. Rev. Lett. 50, 1431 (T983).

27. S.R. Smith et al., Phys. Rev. Lett. 51, 743 (1983). 